\title{
VERIFICATION AND ANALYSIS OF AN IMPROVED AUTHENTICATION PROTOCOL FOR MOBILE IP
}

\author{
Qing Gao \\ Dept of Electrical Engineering \\ Faculty of Engineering \\ National University of Singapore \\ 10 Kent Ridge Crescent \\ Singapore 119260 \\ engp8682@nus.edu.sg
}

\author{
Winston Seah, Anthony Lo, Kin-Mun Lye \\ Centre for Wireless Communications ${ }^{1}$ \\ National University of Singapore \\ 20 Science Park Road \\ \#02-34/37 TeleTech Park \\ Singapore Science Park II \\ Singapore 117674
}

\begin{abstract}
In this paper, we proposed an authentication protocol for Mobile IP to improve its scalability and efficiency. The protocol was verified and analysed with BAN logic. We discovered that there is a tradeoff between security and efficiency. To improve the efficiency, we omitted the direct authentication between the Mobile Host (MH) and the Foreign Agent (FA) which resulted in weaker beliefs. However, through discussion, we were able to show that the result does not compromise the security of the protocol.
\end{abstract}

Keywords: Mobile IP, Scalable Tripartite Authentication, public-key cryptographic mechanism, secret-key cryptographic mechanism, BAN logic.

\section{INTRODUCTION}

In previous work [2], an improved authentication protocol for Mobile IP $[5,7]$ which is based on both the secret-key and the public-key cryptographic mechanism has been proposed. The protocol realizes a scalable tripartite authentication among the Mobile Host (MH), the Foreign Agent (FA) and the Home Agent (HA). Meanwhile, it improves the efficiency as compared to existing scalable protocols. In order to check the security of the protocol, we adopted the widely used BAN Logic [1] to verify the proposed authentication protocol. In the verification process, we found that the result was weaker than the other tripartite authentication schemes based on public key methods [4] or

\footnotetext{
${ }^{1} \mathrm{CWC}$ is a national R \& D Centre funded by the Singapore National Science and Technology Board.
} 
shared key methods [6]. However, we were able to show that the result does not compromise the security of the protocol, and furthermore, our proposed protocol is definitely more computationally efficient and scalable.

This paper is organized as follows. Section 2 presents the improved Mobile IP registration authentication protocol. Section 3 briefly presents the authentication verification logic, BAN logic. Section 4 describes the verification procedure of the proposed authentication protocol. Section 5 analyses the plausible security problem incurred because of the lack of direct MH-FA authentication. Section 6 concludes the paper.

\section{IMPROVED AUTHENTICATION PROTOCOL FOR MOBILE IP}

In [2], we proposed an improved authentication protocol for Mobile IP, as shown in Figure 1, which realizes the tripartite authentication among the $\mathrm{MH}$, the FA and the HA. It improves the security and scalability of Mobile IP but at the same time minimizes computational overheads and re-registration delay as much as possible. In the proposal, we assume that both the $\mathrm{MH}$ and the HA belong to the same administrative domain, and the number of MHs an HA handles is not too large. Therefore, the symmetric key mechanism as in the standard Mobile IP can be used in the MH-HA authentication in order to take advantage of its computational efficiency and simplicity. As the $\mathrm{MH}$ could travel across a considerably large area, neither the HA nor the MH would know how many FAs they have to authenticate. Hence, for scalability reason, we use the public-key cryptographic mechanism for the authentication between the FA and the HA. The MH-FA pair is implicitly authenticated without compromising the security when the authentication between the MH-HA and FA-HA is successfully performed. Also, this minimizes the re-registration delay and further improves the efficiency of the protocol.

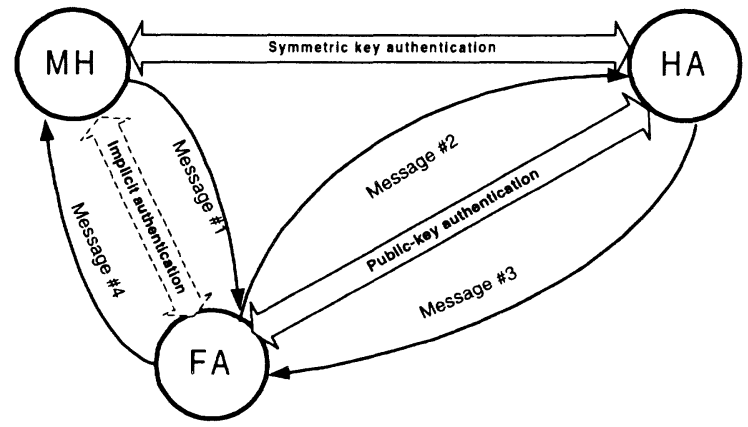

Figure 1 Tripartite two-way authentication protocol 
The steps in the improved authentication protocol are explained below and Table 1 defines the terminologies used in the description.

Table 1: Definitions of the nomenclture used in the authentication protocol $^{a}$

\begin{tabular}{|c|c|}
\hline Nomenclature & Definition \\
\hline$E(X, Y)$ & the encryption of $Y$ under key $X$ \\
\hline $\operatorname{Auth}(X)$ & the message digest function value on contents $X$ \\
\hline Priv FA & the private key of foreign agent \\
\hline Pub_FA & the public key of foreign agent \\
\hline Priv HA & the private key of home agent \\
\hline Pub_HA & the public key of home agent \\
\hline $\operatorname{Sig}(X, Y)$ & the signature of $Y$ with key $X$ where $\operatorname{Sig}(X, Y)=E(X, M D(Y))$. \\
\hline CentFA & certificate of foreign agent \\
\hline Cert_HA & certificate of home agent \\
\hline Pub_CA & public key of the certification authority \\
\hline
\end{tabular}

${ }^{a}$ A certificate is a data structure that binds an entity's identity to a public key with a CA's digital signature.

Step 1: When an $\mathrm{MH}$ detects that it is away from home, it initiates a registration procedure by sending Message \#1 (Figure 2) to the FA.

\begin{tabular}{|c|c|c|}
\hline $\begin{array}{c}\text { Registration } \\
\text { Request }\end{array}$ & ID & Auth(Registration Request, ID, K) \\
\hline
\end{tabular}

Figure 2 Message \#1 MH $\rightarrow$ FA

$\mathrm{K}$ is the secret key shared between the MH and the HA. ID is set as the timestamp or a nonce as described in the basic Mobile IP protocol [5] to perform replay protection. Auth(Registration Request, ID, $\mathrm{K}$ ) is the message digest function value on contents (Registration Request, ID, K).

Step 2: When the FA receives Message \#1, it adds its certificate (Cert_FA) to the message, then signs it and creates Message \#2 (Figure 3). Then the FA relays Message \#2 to the HA. (Note: The unshaded part is the standard Mobile $I P$ registration message expunging the authentication extension. The shaded part contains the fields that differ from the standard protocol, including the new authentication extension.)

\begin{tabular}{|c|c|c|c|c|}
\hline $\begin{array}{c}\text { Registration } \\
\text { Request }\end{array}$ & ID & $\begin{array}{c}\text { Auth(Registration } \\
\text { Request, ID, K) }\end{array}$ & Cert_FA & $\begin{array}{c}\text { Sig\{Priv_FA, (Registration Request, ID, } \\
\text { Auth(Registration Request, ID, K), Cert_FA)\} }\end{array}$ \\
\hline
\end{tabular}

Figure 3 Message \#2 FA $\rightarrow$ HA

Step 3: When the HA receives Message \#2, its actions are shown in Figure 4.

Then the HA sends Message \#3 (Figure 5) to the FA. It indicates whether the MH's registration is accepted or refused as well as the refusal reason. Note that, in Message \#3, HA adds the FA identifier, FA_ID, into the message indicating that it trusts FA. 


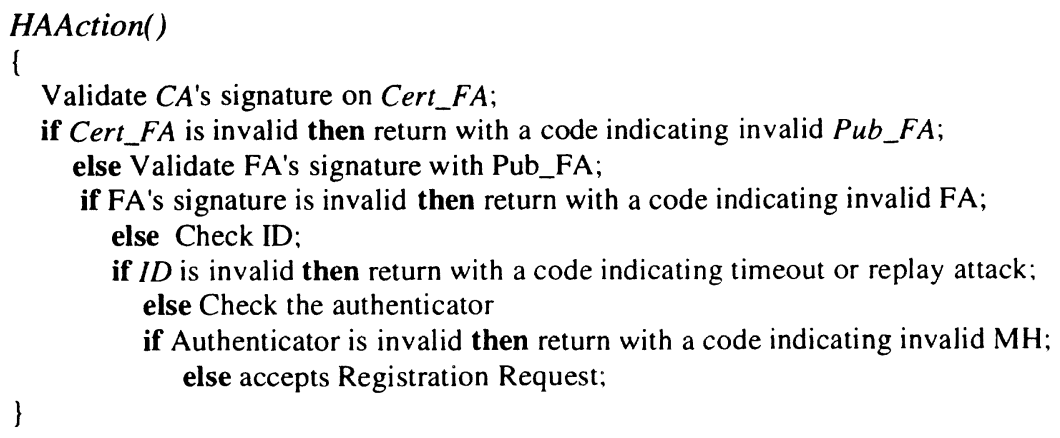

Figure 4 Actions of the HA when receives Message \#2

\begin{tabular}{|c|c|c|c|c|c|}
\hline $\begin{array}{c}\text { Registration } \\
\text { Reply }\end{array}$ & ID & FA_ID & $\begin{array}{c}\text { Auth(Registration } \\
\text { Reply, ID,FA_ID, K) }\end{array}$ & Cert_HA & $\begin{array}{c}\text { Sig\{Priv_HA, (Registration Reply, ID, FA_ID } \\
\text { Auth(Registration Reply, ID,FA_ID, K), Cert_HA)\} }\end{array}$ \\
\hline
\end{tabular}

Figure 5 Message \#3 HA $\rightarrow$ FA

Step 4: When the FA receives Message \#3, its actions are shown in Figure 6.

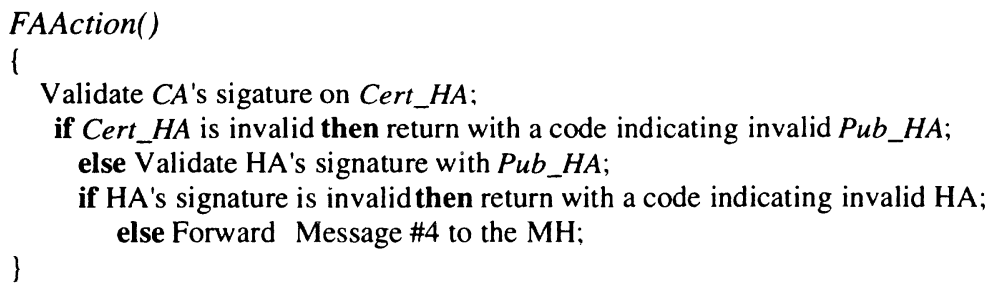

Figure 6 Actions of the FA when receives Message \#3

If $\mathrm{HA}$ is valid, no matter what the contents in the Registration Reply is, FA will relay the first half of Message \#3, i.e., Message \#4 (Figure 7).

\begin{tabular}{|c|c|c|c|}
\hline $\begin{array}{c}\text { Registration } \\
\text { Reply }\end{array}$ & ID & FA_ID & Auth(Registration Reply, ID, FA_ID, K) \\
\hline
\end{tabular}

Figure 7 Message \#4 FA $\rightarrow$ MH

Step 5: When the $\mathrm{MH}$ receives Message \#4, its actions are shown in Figure 8.

\section{OUTLINE OF THE VERIFICATION METHODOLOGY BASED ON BAN LOGIC \\ 3.1 BAN LOGIC}

BAN logic [1] is a widely used logic specifically designed for analyzing the protocols for the authentication of principals in distributed computing systems. 


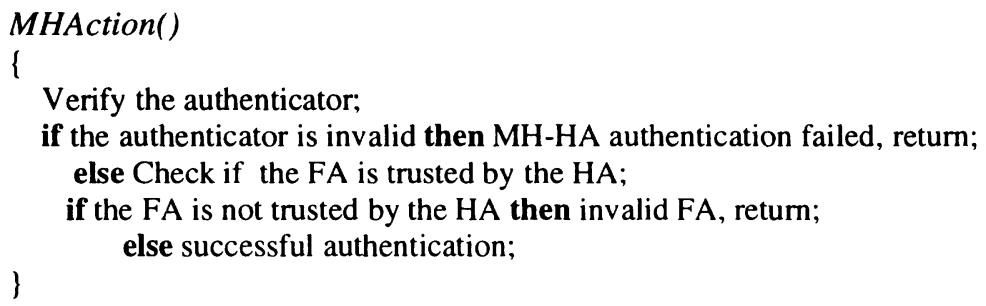

Figure 8 Actions of the $\mathrm{MH}$ when receives Message \#4

We use this logic to verify the improved authentication protocol for Mobile IP [2].

To use BAN logic for verification, one has to formalize the protocol with the BAN notations. The reason is that the conventional notations for describing an authentication protocol is not convenient for manipulation in a logic since the contents of each message often means more than necessary in the security sense. With the BAN notations, one can transform each message into a logical formula which is an idealized version of the original message. The notations shown in Table 2 will be used throughout the paper.

BAN logic uses some logical postulates to annotate an idealized protocol with assertions. An assertion usually describes beliefs held by the principals at the point in the protocol where the assertion is inserted. An annotation consists of a sequence of assertions inserted before the first statement and after each statement. To express that the statement $Z$ follows from a conjunction of statements $X$ and $Y$, we write: $\frac{X, Y}{Z}$. The postulates are listed as follows:

- The message-meaning rules:

For shared keys:

Rule 1: $\frac{P\left\lfloor\equiv P \stackrel{K}{\leftrightarrow} Q, P \triangleleft\{X\}_{K}\right.}{P|\equiv Q| \sim X}$ (That is, if $P$ believes that the key $K$ is shared with $Q$ and sees a message $X$ encrypted under $K$, then $P$ believes that $Q$ once sent $X$.)

Rule 2: $\frac{P \mid \equiv Q \stackrel{K}{\rightleftharpoons} Q, P \triangleleft\langle X\rangle_{Y}}{P|\equiv Q| \sim X}$ (That is, if $P$ believes that the secret $Y$ is shared with $Q$ and sees $\langle X\rangle_{Y}$, then $P$ believes that $Q$ once sent $X$.)

For public keys:

$\frac{P \mid \equiv \stackrel{K}{\mapsto} Q, P \triangleleft\{X\}_{K-1}}{P|\equiv Q| \sim X}$ (That is, if $P$ believes that the key $K$ is the public key of $Q$ and sees a message $X$ encrypted under $Q$ 's private key $K^{-1}$, then $P$ believes that $Q$ once sent $X$.)

- The nonce-verification rule:

$\frac{P|\equiv \#(X), P| \equiv Q \mid \sim X}{P|\equiv Q| \equiv X}$ (That is, if $P$ believes that $X$ could have been uttered only recently and that $Q$ once sent $X$, then $P$ believes that $Q$ believes $X$.)

- The jurisdiction rule: 
Table 2: Notations in BAN logic

\begin{tabular}{|c|c|}
\hline Notation & Definition \\
\hline$A, B, S$ & specific principals \\
\hline$K_{a b}, K_{a s}, K_{b s}$ & specific shared keys \\
\hline$K_{a}, K_{b}, K_{s}$ & specific public keys \\
\hline$N_{a}, N_{b}$ & specific statements \\
\hline$P, Q$ & general principals \\
\hline$X, Y$ & general statements \\
\hline$K$ & $P$ is persuaded of the truth of $X$ \\
\hline$P \mid \equiv X$ & $P$ receives a message containing $X$ \\
\hline$P \triangleleft X$ & $P$ has jurisdiction over $X$,or \\
\hline$P \mid \sim X$ & $P$ is trusted as an authority on $X$ \\
\hline$P \Rightarrow X$ & a previous run of the protocol \\
\hline$\#(X)$ & $P$ and $Q$ have a shared key $K$ \\
\hline$P \stackrel{K}{\leftrightarrow} Q$ & $X$ has not been sent in a message belong to \\
\hline$\mapsto P$ & $P$ has $K$ as a public key and $K^{-1}$ as a private key \\
\hline$P \stackrel{X}{\rightleftharpoons} Q$ & $X$ is a secret known only to $P$ and $Q$ \\
\hline$\{X\}_{K}$ & $X$ is encrypted under the key $K$ \\
\hline$\langle X\rangle_{Y}$ & $X$ is combined with $Y ; Y$ is a secret whose \\
& presence proves the identity of whoever utters $\langle X\rangle_{Y}$ \\
\hline
\end{tabular}

$\frac{P|\equiv Q \Rightarrow X, P| \equiv Q \mid \equiv X}{P \mid \equiv X}$ (That is, if $P$ believes that $Q$ has jurisdiction over $X$ and that $P$ believes that $Q$ believes $X$, then $P$ trusts $Q$ on the truth of $X$.)

The assertions are expressed in the same notations used to write messages. Moreover, annotations can be concatenated and new assertions can be derived from established ones. For example, if the $X$ is an assertion in a legal annotation $A$, if $X^{\prime}$ is provable from $X$, and if $A^{\prime}$ is the result of substituting $X^{\prime}$ for $X$ in $A$, then $A^{\prime}$ is a legal annotation.

The desired goals of an authentication protocol can be specified in BAN logic. Usually, for secret key authentication protocols, we might deem that an authentication is complete between $A$ and $B$ if there is a $K$ such that:

$A \mid \equiv A \stackrel{K}{\leftrightarrow} B$ ( $A$ believes that $A$ and $B$ has a shared key $K$.)

$B \mid \equiv A \stackrel{K}{\leftrightarrow} B(B$ believes that $A$ and $B$ has a shared key $K$.)

Or in addition:

$A|\equiv B| \equiv A \leftrightarrow B$ (A believes that $B$ believes that $A$ and $B$ has a shared key $K$.) 
$B|\equiv A| \equiv A \stackrel{K}{\leftrightarrow} B$ ( $B$ believes that $A$ believes that $A$ and $B$ has a shared key $K$.)

Or even weaker goals.

For public-key authentication protocols, the goal may be:

$A \mid \equiv \stackrel{K}{\mapsto} B$ ( $A$ believes that $B$ has $K$ as a public key.)

Or in addition: $\quad A \mid \equiv A \stackrel{N_{a}}{\rightleftharpoons} B\left(A\right.$ believes that $A$ and $B$ has a secret $N_{a}$.)

\subsection{THE VERIFICATION METHODOLOGY}

The verification methodology is outlined as follows:

1. Define the original authentication protocol with the conventional notations.

2. Formalize the authentication goals using BAN logic notations.

3. Derive the idealized protocol from the original one. This generally involves simplifying the protocol by omitting the parts of the message that do not contribute to the beliefs of the recipient. The idealized protocols do not include cleartext message parts because it can be easily forged.

4. Formalize the assumptions about the initial state with BAN logic notations.

5. Verify the protocol. This includes attaching the logical formulas to the statements of the protocol as assertions about the state of the system and applying the logical postulates to the assumptions and the assertions until the beliefs held by the parties in the protocol is deduced.

\section{VERIFICATION OF THE IMPROVED AUTHENTICATION PROTOCOL USING BAN LOGIC}

\subsection{ORIGINAL PROTOCOL}

The following notations are defined to be used in the verification procedure.
S: CA
$A: \mathrm{MH}$
$B$ : FA
$C:$ HA

$X_{a}$ : Registration Request produced by $A(\mathrm{MH})$

$X_{c}$ : Registration Reply produced by $C(\mathrm{HA})$

$T_{a}: I D \_$RegRequest (ID in Figure 2) $T_{c}: I D \_R e g R e p l y$ (ID in Figure 5)

$K_{a c}$ : the key shared between $\mathrm{MH}$ and HA ( $K$ in Figure 2,3,5,7)

$K_{b}, K_{c}, K_{s}$ : the respective public keys of FA, HA and CA

$K_{b}^{-1}, K_{c}^{-1}, K_{s}^{-1}$ : the respective private keys of FA, HA and CA 
The messages in the improved authentication protocol as described in Section 2 are defined in the conventional notations as follows:

Message \#1. $A \longrightarrow B:\left\{X_{a}, T_{a},\left\{X_{a}, T_{a}, K_{a c}\right\}_{K_{a c}}\right\}$

(See also Figure 2, $\left\{X_{a}, T_{a}, K_{a c}\right\}_{K_{a c}}$ corresponds to Auth(Registration Request, ID. K).)

Message \#2. $B \rightarrow C:\left\{\begin{array}{c}\left\{X_{a}, T_{a},\left\{X_{a}, T_{a}, K_{a c}\right\}_{K_{a c}}\right\},\left\{B, K_{b}\right\}_{K_{s}^{-1}}, \\ \left.\left\{X_{a}, T_{a},\left\{X_{a}, T_{a}, K_{a c}\right\}_{K_{a c}}\right\},\left\{B, K_{b}\right\}_{K_{s}^{-1}},\right\}_{K_{b}^{-1}}\end{array}\right\}$

(See also Figure 3, $\left\{B, K_{b}\right\}_{K_{s}^{-1}}$ corresponds to Cert_FA, the last term corresponds to FA's signature.)

Message \#3. $C \longrightarrow B:\left\{\begin{array}{c}X_{c}, T_{c}, B,\left\{X_{c}, T_{c}, B, K_{a c}\right\}_{K_{a c}},\left\{C, K_{c}\right\}_{K_{s}^{-1}}, \\ \left.\left\{X_{c}, T_{c},\left\{X_{a}, T_{c}, B, K_{a c}\right\}_{K_{a c}}\right\},\left\{C, K_{c}\right\}_{K_{s}^{-1}},\right\}_{K_{c}^{-1}}\end{array}\right\}$

(See also Figure 5, $\left\{X_{c}, T_{c}, B, K_{a c}\right\}_{K_{a c}}$ corresponds to Auth(Registration Reply, ID, FAJD, K), $\left\{C, K_{c}\right\}_{K_{s}^{-1}}$ corresponds to Cert_HA, the last term corresponds to HA's signature.)

Message \#4. $B \longrightarrow A:\left\{X_{c}, T_{c}, B,\left\{X_{c}, T_{c}, B, K_{a c}\right\}_{K_{a c}}\right\}$

(See also Figure 6.)

\subsection{AUTHENTICATION GOALS}

The goals that need to be achieved by the tripartite authentication protocol can be deduced from $[1,3,4,5]$. Through authentication, the HA would know whether the Registration Request message it received is really produced by the $\mathrm{MH}$ if it can derive that the MH has the right key shared with itself and the Registration Request is freshly operated by the very key. Similarly, the $\mathrm{MH}$ would know whether the Registration Reply message it received is really produced by the HA if it can derive that the HA has the right key shared with itself and the Registration Reply is freshly operated by the very key. Moreover, the HA would know whether the FA is the authentic one by testing its certificate and its signature. It is the same with the FA. Finally, the MH would also know whether the FA is the authentic one and the FA would know whether the MH is a legal host. The goals are listed as follows with BAN logic notations:

(1) $A|\equiv C| \equiv A \stackrel{K_{\text {ac }}}{\leftrightarrow} C$ (A believes that $C$ believes the shared key $K_{a c}$ between $A$ and $C$ )

(2) $A|\equiv C| \equiv X_{c}$ (A believes that $C$ really sends the timely $X_{c}$ )

(3) $C|\equiv A| \equiv A \stackrel{K_{q c}}{\leftrightarrow} C$ (C believes that $A$ believes the shared key $K_{a c}$ between $A$ and $\left.C\right)$

(4) $C|\equiv A| \equiv X_{a}$ (C believes that $A$ really sends the timely $X_{a}$ )

(5) $B \mid \equiv \stackrel{K_{c}}{\mapsto} C$ (B believes that $K_{c}$ is the public key of $C$ )

(6) $B|\equiv C| \equiv\left\{X_{c}, T_{c}, B, A \stackrel{K_{a c}}{\leftrightarrow} C\right\}_{K_{a c}}$ (B believes that $C$ really sends the timely $\left\{X_{c}, T_{c}, B, A \stackrel{K_{a c}}{\leftrightarrow} C\right\}_{K_{a c}}$,

(7) $C \mid \equiv \stackrel{K_{b}}{\mapsto} B\left(C\right.$ believes that $K_{b}$ is the public key of $\left.B\right)$ 
(8) $C|\equiv B| \equiv\left\{X_{a}, T_{a}, A \stackrel{K_{g c}}{\leftrightarrow} C\right\}_{K_{a c}} \quad(C$ believes that $B$ really sends the timely $\left\{X_{a}, T_{a}, A \stackrel{K_{g c}}{\leftrightarrow} C\right\}_{K_{a c}}$,

(9) $A \mid \equiv B(A$ believes the identity of $B)$

(10) $B \mid \equiv A(B$ believes the identity of $A)$

\subsection{IDEALIZED PROTOCOL}

The original protocol messages are transformed into the idealized form using BAN logic notations.

Message \#1. (Cleartext, omitted)

Message \#2. $B \longrightarrow C$ :

$\left\{X_{a}, T_{a}, A \stackrel{K_{a c}}{\leftrightarrow} C\right\}_{K_{a c}},\left\{B, K_{b}\right\}_{K_{s}^{-1}},\left\{\begin{array}{c}\left\{X_{a}, T_{a},\left\{X_{a}, T_{a}, A \stackrel{K_{a c}}{\leftrightarrow} C\right\}_{K_{a c}}\right. \\ \left\{B, K_{b}\right\}_{K_{s}^{-1}}\end{array}\right\}_{K_{b}^{-1}}$

Message \#3. $C \longrightarrow B$ :

$\left\{X_{c}, T_{c}, B, A \stackrel{K_{a c}}{\leftrightarrow} C\right\}_{K_{a c}},\left\{C, K_{c}\right\}_{K_{s}^{-1}},\left\{\left\{X_{c}, T_{c},\left\{X_{c}, T_{c}, B, A \stackrel{K_{g c}}{\leftrightarrow} C\right\}_{K_{a c}}\right\},\right\}_{K_{c}^{-1}}$

Message \#4. $B \longrightarrow A:\left\{X_{c}, T_{c}, B, A \stackrel{K_{a c}}{\leftrightarrow} C\right\}_{K_{a c}}$

\subsection{ASSUMPTIONS}

We formalize the assumptions for the improved authentication protocol with BAN logic notations as in Table 3:

Table 3: Improved Authentication Protocol Assumptions

\begin{tabular}{|c|c|c|}
\hline A believes: & $B$ believes: & C believes: \\
\hline$A \stackrel{K g^{c}}{\leftrightarrow} C(1)$ & $\stackrel{K_{b}}{\mapsto} B$ & $\stackrel{K_{f}}{\mapsto} C(3)$ \\
\hline$\#\left(T_{a}\right)$ & $\#\left(T_{a}\right)$ & $\#\left(T_{a}\right)(6)$ \\
\hline \multirow[t]{4}{*}{$\#\left(T_{c}\right)$} & $\#\left(T_{c}\right)$ & $\#\left(T_{c}\right)(9)$ \\
\hline & $\stackrel{K_{s}}{\mapsto} S$ & $\stackrel{K_{s}}{\mapsto} S(11)$ \\
\hline & $S \Rightarrow \stackrel{K_{f}}{\mapsto} C(12)$ & $S \Rightarrow \stackrel{K_{b}}{\mapsto} B(13)$ \\
\hline & $S \mid \equiv K_{f} C(14)$ & 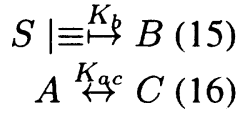 \\
\hline
\end{tabular}




\subsection{VERIFICATION}

According to the rule of jurisdiction, with assumption (13) and (15) listed in Table 3, we get: $\frac{C\left|\equiv\left(S \Rightarrow \stackrel{K_{b}}{\hookrightarrow} B\right), C\right| \equiv\left(S \mid \equiv{ }_{K_{b}} B\right)}{C \mid \equiv{ }_{b} B}$ (Accords to goal 7)

Thus, by the message meaning rule for public keys and message \#2, we get: $\frac{C \mid \equiv \stackrel{K_{b}}{\mapsto} B, C \triangleleft\left\{\left\{X_{a}, T_{a}, A \stackrel{K_{a c}}{\leftrightarrow} C\right\}_{K_{a c}}\right\}_{K_{b}^{-1}}}{C \mid \equiv\left(B \mid \sim\left\{X_{a}, T_{a}, A \stackrel{K_{a c}}{\leftrightarrow} C\right\}_{K_{a c}}\right)}$

Thus, by the nonce verification rule and assumption (6), we get: $\frac{C\left|\equiv \#\left(T_{a}\right), C\right| \equiv\left(B \mid \sim\left\{X_{a}, T_{a}, A{ }^{K_{a c}} C\right\}_{\left.K_{a c}\right)}\right)}{C \mid \equiv\left(B \mid \equiv\left\{X_{a}, T_{a}, A_{a q}^{K_{a c}} C\right\}_{K_{a c}}\right)} \ldots \ldots \ldots \ldots \ldots \ldots \ldots$ (Accords to goal 8) Since message \#2 contains $T_{a}$, we can derive: $C \mid \equiv \#\left(X_{a}\right)$

By the message meaning rule (1) for shared keys and assumption (16), we get: $\frac{C \mid \equiv A \stackrel{K_{a c} c}{\leftrightarrow}, C \triangleleft\left\{X_{a}, T_{a}, A \stackrel{K_{a c}}{\leftrightarrow} C\right\}_{K_{a c}}}{C \mid \equiv\left(A \mid \sim X_{a}\right)}$

Thus, according to the nonce verification rule, we get: $\frac{C\left|\equiv \#\left(X_{a}\right), C\right| \equiv\left(A \mid \sim X_{a}\right)}{C \mid \equiv\left(A \mid \equiv X_{a}\right)}$ (Accords to goal 4)

By the message meaning rule (1) for shared keys, with assumption (16) and message \#2, we get: $\frac{C \mid \equiv A \stackrel{K_{a c}}{\leftrightarrow} C, C \triangleleft\left\{X_{a}, T_{a}, A \stackrel{K_{a c}}{\leftrightarrow} C\right\}_{K_{a c}}}{C \mid \equiv\left(A \mid \sim A \stackrel{K_{a c}}{\leftrightarrow} C\right)}$

Since message \#2 contains $T_{a}$, we can derive: $C \equiv \#\left(A \stackrel{K_{\sharp c}}{\leftrightarrow} C\right)$ Thus, by the nonce verification rule, we get: $\frac{C\left|\equiv \#\left(A \stackrel{K_{a c}}{\leftrightarrow} C\right), C\right| \equiv\left(A \mid \sim A \stackrel{K_{a c}}{\leftrightarrow} C\right)}{C \mid \equiv\left(A \mid \equiv A \stackrel{K_{a c}}{\leftrightarrow} C\right)}$ (Accords to goal 3)

According to assumption (12) and message \#3, by the rule of jurisdicton, we get: $\frac{B\left|\equiv\left(S \Rightarrow \stackrel{K_{\digamma}}{\mapsto} C\right), B\right| \equiv\left(S \mid \equiv \stackrel{K_{\digamma}}{\hookrightarrow} C\right)}{B \mid \equiv \stackrel{K_{f}}{\lessgtr} C}$ (Accords to goal 5)

Thus, by the message meaning rule for public keys and message \#3, we get: $\frac{B \mid \equiv \stackrel{K_{\xi}}{\mapsto} C, B \triangleleft\left\{\left\{X_{c}, T_{c}, B, A \stackrel{K_{q c}}{\leftrightarrow} C\right\}_{K_{a c}}, X_{c}\right\}_{K_{c}^{-1}}}{B \mid \equiv\left(C \mid \sim\left\{\left\{X_{c}, T_{c}, B, A \stackrel{K_{q c}}{\leftrightarrow} C\right\}_{K_{a c}}, X_{c}\right\}\right)}$

Since message \#3 contains $T_{c}$, we can derive: $B \mid \equiv \#\left(\left\{X_{c}, T_{c}, B, A \stackrel{K_{g c}}{\leftrightarrow}\right.\right.$ $C\}_{K_{a c}}, X_{c}$ )

Thus, by the nonce verification rule, we get:

$$
\frac{B\left|\equiv \#\left(\left\{X_{c}, T_{c}, B, A \stackrel{K_{a c}}{\leftrightarrow} C\right\}_{K_{a c}}\right), B\right| \equiv\left(C \mid \sim\left\{X_{c}, T_{c}, B, A \stackrel{K_{a c}}{\leftrightarrow} C\right\}_{K_{a c}}\right)}{B \mid \equiv\left(C \mid \equiv\left\{X_{c}, T_{c}, B, A \stackrel{K_{\leftrightarrow c} C}{\leftrightarrow} C\right\}_{K_{a c}}\right)}
$$
(Accords to goal 6)

By the message meaning rule for public keys, we get: $\frac{B \mid \equiv{ }_{c}^{K_{c}} C, B \triangleleft\left\{X_{c}\right\}_{K_{c}^{-1}}}{B \mid \equiv\left(C \mid \sim X_{c}\right)}$ Moreover, by the nonce verification rule, we get: $\frac{B\left|\equiv \#\left(X_{c}\right), B\right| \equiv\left(C \mid \sim X_{c}\right)}{B \mid \equiv\left(C \mid \equiv X_{c}\right)}$ From the content of $X_{c}$, B knows if $\mathrm{C}$ has refused $\mathrm{A}$ because of authentication failure. If not, $\mathrm{B}$ knows that $\mathrm{A}$ is trusted by $\mathrm{C}$, thus we derive the new assertions: $B \mid \equiv\left(C \mid \equiv\left(A \mid \equiv A \stackrel{K_{a c}}{\leftrightarrow} C\right)\right)\left(B\right.$ believes that $C$ believes that $A$ really have the key, $K_{a c}$, 
shared between $A$ and $C$ ) (Weaker than goal 10)

and then go to message \#4; otherwise, B stops here.

Since message \#4 contains $T_{c}$, we can derive: $A \mid \equiv \#\left(A \stackrel{K_{q c}}{\leftrightarrow} C, X_{c}, B\right)$ By the message meaning rule (1) for shared keys and assumption (1), we get: $\frac{A \mid \equiv A \stackrel{K_{a c}}{\leftrightarrow} C, A \triangleleft\left\{X_{c}, B\right\}_{K_{a c}}}{A \mid \equiv\left(C \mid \sim\left\{X_{c}, B\right\}\right)}$

Thus, according to the nonce verification rule, we get:

$\frac{A\left|\equiv \#\left(X_{c}\right), A\right| \equiv\left(C \mid \sim X_{c}\right)}{A \mid \equiv\left(C \mid \equiv X_{c}\right)}$ (Accords to goal 2)

According to message \#4 and assumption (1), by the message meaning rule (1) for shared keys, we get: $\frac{A \mid \equiv A \stackrel{K_{a c}}{\leftrightarrow} C, A \triangleleft\left\{X_{c}, T_{c}, B, A \stackrel{K_{a c}}{\leftrightarrow} C\right\}_{K_{a c}}}{A \mid \equiv\left(C \mid \sim A \stackrel{K_{a c} c}{\leftrightarrow} C\right)}$

Thus, according to the nonce verification rule, we get:

$\frac{A\left|\equiv \#\left(A \stackrel{K_{a c}}{\leftrightarrow} C\right), A\right| \equiv\left(C \mid \sim A \stackrel{K_{a c}}{\leftrightarrow} C\right)}{A \mid \equiv\left(C \mid \equiv A_{\leftrightarrow a c}^{K_{\hookrightarrow} c} C\right)}$ (Accords to goal 1 )

Finally, by the nonce verification rule, we get:

$\frac{A|\equiv \#(B), A| \equiv(C \mid \sim B)}{A \mid \equiv(C \mid \equiv B)}$ ( $A$ believes that $C$ believes the identity of $\left.B\right)$. (Weaker than goal 9 )

\section{DISCUSSION}

From the verification results above, we achieved all of the authentication goals except goals (9) and (10). That is, the results showed that the MH and HA trust each other and the FA and HA trust each other. Since the direct authentication between the $\mathrm{MH}$ and the FA was omitted, we could not derive that the MH and FA trust each other, but that the MH trusts that the HA trusts the FA and the FA trusts that the HA trusts the MH. However, they can be viewed as having implicitly authenticated each other. The result was weaker than the other schemes $[3,4,6]$ that perform direct authentication between the $\mathrm{MH}$ and FA, but we will show that it is enough for a secure registration process. In other words, the weaker beliefs do not compromise the security of the protocol, which we explain as follows.

While an illegal FA, say $F A_{i l}$, masquerades as a legal one, say $F A_{l}$, it will fail to authenticate its identity to the HA. As we know, $F A_{i l}$ does not have $F A_{l}$ 's private key, so the HA will send back a Registration Reply with a code indicating that the FA failed authentication. Then the MH will try to find another FA. If $F A_{i l}$ concocts a Registration Reply and transfers it to the $\mathrm{MH}$ instead of the original Registration Reply, the $\mathrm{MH}$ will also find the truth since $F A_{i l}$ cannot concoct the right message digest which is supposed to compute with the key shared between the MH and HA. In such a case, the MH will try to find another FA. Note that, in this protocol, as long as an FA is not trusted at first, what it sends to the MH as Registration Reply will be ignored by the $\mathrm{MH}$. That is, we deprived the right for the FA to refuse the $\mathrm{MH}$, thus getting rid of the probability that an illegal FA performs denial-of-service attack by 
issuing Registration Reply stating the $\mathrm{MH}$ is denied. Meanwhile, when the $\mathrm{MH}$ received no reply within the timeout period and it has retransmitted the Registration Reply $\mathrm{n}$ times ( $\mathrm{n}$ is a predesigned number of retransmission) in vain, the $\mathrm{MH}$ should intelligently try to find another FA.

Likewise, while an illegal $\mathrm{MH}$, say $M H_{i l}$, masquerades as a legal one, say $M H_{l}$, it will fail to authenticate its identity to the HA because it does not have the key shared between the HA and $M H_{l}$. Hence, the FA will know it from message \#3 in which contained a code indicating that the $\mathrm{MH}$ failed authentication, so that the FA will not trust this $M H_{i l}$.

In summary, our protocol meets the need for a secure Mobile IP authentication, i.e., it realizes the tripartite authentication.

\section{CONCLUSIONS}

This paper verified and analyzed the security of an improved authentication protocol for Mobile IP, which realizes an efficient and scalable tripartite authentication among the MH, the FA and the HA. The widely used BAN logic was used in the verification process. The result of the verification showed that desired beliefs were achieved for the MH-HA pair and the HA-FA pair. However, weaker beliefs were resulted from the $\mathrm{MH}$ and FA pair since we omitted the direct authentication between them. However, we showed that the weakness does not compromise the security of the protocol. Therefore, the proposed improved authentication protocol meets the security requirement of Mobile IP.

\section{References}

[1] Burrows M., Abadi, M., Needham R., (1990). "A Logic of Authentication". http://www.research.digital.com/SRC/personal/Martin_Abadi/Papers/src39revised.ps.

[2] Gao, Q., Seah W., Lo A., (1999). "Secure and Improved Authentication in Mobile IP ". Technical report 1999-NETWORKS-TR-001NR, Centre for Wireless Communications.

[3] Jacobs, S., (1998). "Mobile IP Public Key Based Authentication". draftjacobs-mobileip-pki-auth-00.txt (work in progress).

[4] Jacobs, S., (1999). "Mobile IP Public Key Based Authentication". draftjacobs-mobileip-pki-auth-01.txt (work in progress).

[5] Perkins, C., (1996). "IP Mobility Support". RFC2002.

[6] Sanchez,L. A. and Troxel, G. D., (1997). "Rapid Authentication for Mobile IP". draft-ietf-mobileip-ra-00.txt (work in progress).

[7] Solomon, J. D., (1998). Mobile IP, the Internet unplugged. Upper Saddle River, N.J. : PTR Prentice Hall. 